Dear author,

Please note that changes made in the online proofing system will be added to the article before publication but are not reflected in this PDF.

We also ask that this file not be used for submitting corrections. 


\section{c0085 Application of Scanning Electron Microscopy-Energy Dispersive X-Ray Spectroscopy (SEM-EDS)}

\section{A.V. Girão, ${ }^{*}{ }^{1}$ G. Caputo ${ }^{\S}$ and M.C. Ferro*}

*CICECO and Department of Materials and Ceramic Engineering, University of Aveiro,

[Q1] Campus de Santiago, Aveiro, Portugal; ${ }^{\S}$ Istituto Italiano Di Tecnologia, Genova, Italy

${ }^{1}$ Corresponding author: E-mail: avgirao@ua.pt

\section{Chapter Outline}

1. Introduction

1.1 Scanning Electron Microscopy

1.2 Energy Dispersive X-Ray Spectroscopy
2 2. Sample Preparation 6

3. Equipment Operation 9

3 4. Microplastics Characterization 12

5. Final Considerations 14

6 References 14

List of Abbreviations

AFM Atomic force microscopy

CPS counts per second

EDS Energy X-ray dispersion spectroscopy

EM Electron microscopy

ESEM Environmental scanning electron microscopy

LEDs Light emitting diodes

OM Optical-light microscopy

PE Polyethylene
PMMA Polymethylmethacrylate

SEM Scanning electron microscopy

PP Polypropylene

PS Polystyrene

PVC Polyvinyl chloride

TEM Transmission electron microscopy

UV Ultraviolet

ZAF Atomic number-Z, absorption-A and fluorescence-F 


\section{S0010 1. INTRODUCTION}

p0010 Microscopy is an imaging technique and an essential tool in life and materials science. Generally, the optical microscope transforms an object into an image, and then its true value and excitement subsist in its ability to transform objects outside the range of the unaided eye resolution into much larger images. The endeavour of any microscopy developer and contributor is always to observe smaller objects and create better magnified images. This leads to continuous and countless technological advances with modern microscopy being used for many different purposes and providing fundamental scientific information.

p0015 For centuries, optical-light microscopy (OM) was the only imaging system commonly used in science. It is a simple system in which the object of interest is illuminated by a transmitted light source such as a halogen lamp, lasers or LEDs. A condenser lens then focuses and densifies the light into a more intense one. The latter reaches the specimen fixed onto a mechanical stage that enables movement under a revolving nosepiece which holds several lenses, the objective lenses with different augmentations. The objective lens collects the light and a focused real image is formed. The real image is again enlarged by the ocular lenses giving the final magnified image of the observed specimen. The final image magnification results from the product of the powers of the ocular and objective lenses [1-4].

p0020 OM has several limitations, and the most important one is the loss of resolution at very high magnifications. By applying different physical principles in image generation, several other categories of microscopy have been developed to overcome those restrictions. The most important, electron microscopy (EM), is widely used in materials research as well as in life sciences, and the main modification is the illumination source which is a highly energetic electron beam. The concept is easy to understand if we remember that light as radiation has a wavelength of $400-700 \mathrm{~nm}$ and electrons wavelengths between 0.001 and $0.01 \mathrm{~nm}$. Accordingly, EM provides a highly energetic source of illumination enabling a theoretical resolution of around $0.02 \mathrm{~nm}$, for $100 \mathrm{kV}$ electrons. As any other technique imaging, EM has also some drawbacks such as lens aberration and resolution limits. In due course, one of the main motivations in the improvement of EM is to straightforwardly attain subatomic imaging resolution and elemental analysis [4-6].

p0025 Nowadays, the occurrence of microplastics in the environment needs to be properly addressed as well as their ecological effects after their release into aquatic systems, terrestrial media and atmosphere. The characterization of

[Q2] such microparticles progressed over the past few years, and optical microscopy was one of the first tools used for visual inspection and determination of their shape and size. The amount of identifiable particles by $\mathrm{OM}$ is restricted due to its spatial resolution, whereas, such undetected particles can easily be observed under the electron microscope, enabling accurate size distribution and morphology evaluation. OM and EM are used for very different purposes 
and often complement each other as essential tools in the detection and characterization of microplastics. Nonetheless, EM is one of the few imaging and analytical techniques able to cope with the urgent need for the characterization of microplastics as well as other potentially toxic substances present in the environment which may be adsorbed by the plastic microparticles.

p0030 This chapter demonstrates the increasing importance of scanning electron microscopy (SEM) with coupled energy X-ray dispersion spectroscopy (EDS) as essential tools in the characterization of microplastics. The next sections describe the basic principles of SEM and EDS, followed by a brief discussion on sample preparation and equipment operation. An outline summarizing the effective application of SEM-EDS in the characterization of microplastics is also provided.

\section{s0015 1.1 Scanning Electron Microscopy}

p0035 A scanning electron microscope (SEM) presents a higher complexity level than an optical microscope, as shown in Fig. 1, which schematically depicts the OM and the SEM optical systems.

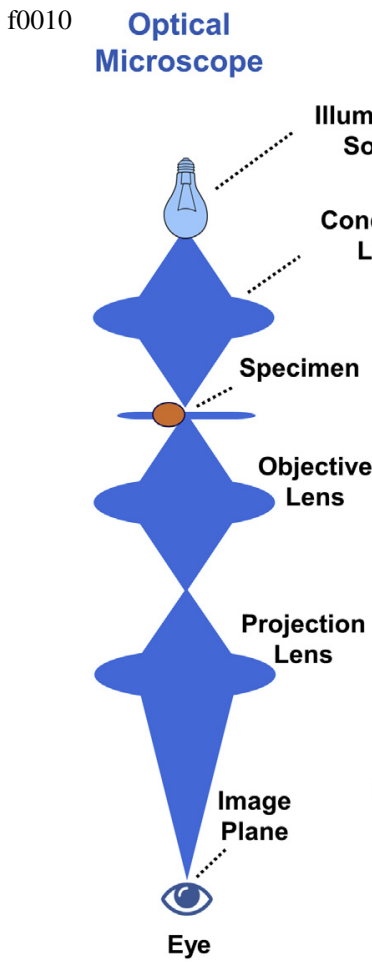

\section{SEM}

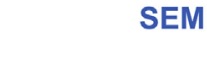


p0040 In SEM, a highly energetic and focused electron beam is formed and scans the specimen in a raster scan pattern. Then, different effects can result from the interaction between the electron beam and the electrons in the sample. Part of the electron beam will be unscattered, though most of the electron beam interacts with the specimen and undergoes inelastic and elastic scattering. In the first case, the direction of the primary electrons is changed but their overall energy is kept. Inelastic scattered electrons change their direction and lose part of their energy. Although most of the electron beam energy ends up in the specimen as heat, other events take place and are detected outside the specimen: secondary effects. Subsequent effects can include emitted secondary electrons, backscattered electrons and characteristic X-rays. The secondary electrons are generated when an incident electron changes its path and loses part of its energy, which is transferred to an atom in the specimen (inelastic event) and leaves the sample with a very small energy. Each primary or incident electron can produce several secondary electrons, thus secondary electrons are abundant and the most used imaging signal in SEM. Backscattered electrons result from the collision between an incident electron and an atom in the specimen and losing part of its energy and being scattered 'backwards' 180 degrees. Some of the backscattered electrons can generate more secondary electrons when exiting the specimen. The production of backscattered electrons varies directly with the atomic number of the chemical elements present in the specimen: the higher the atomic number, the brighter that region will appear. X-rays emission results from the deenergization of an atom in the sample after a secondary electron is produced. This signal is extremely important for analytical purposes, as it will be addressed in the next section. A key factor in SEM is the interaction volume, the region into which the electron beam penetrates the specimen, i.e., the three-dimensional volume resulting from the interaction of the electron beam with the specimen atoms. The emission depth of the different signals used in SEM is influenced by the electron beam energy, specimen nature, composition and sample preparation. The higher the accelerating voltage applied on the electron beam, the larger the interaction volume. In SEM, imaging is carried out by using the emission of secondary electrons (topography) and backscattered electrons (atomic number). Analytical X-rays enable qualitative and quantitative analysis of the specimens. Thus, SEM provides information on the topography, morphology, composition and crystallographic nature of the analysed specimens.

p0045 Though electron and optical microscopes share some common principles, the differences are rather evident as shown in Table 1.

p0050 SEM offers a great deal of information and surpasses most of the limitations of optical microscopy. For samples highly sensitive to the electron beam such as biological samples or synthetic organic polymer materials, there are two alternatives to the conventional SEM, the low-voltage microscope and the environmental SEM (ESEM). The former enables imaging down to a few tens of volts with fairly high resolution proving to be particularly useful for charge 
TABLE 1 Summary of the Main Differences Between Optical and Electron Microscopy [1-6]

Optical-light Microscopy $\quad$ Scanning Electron Microscopy

Illumination source

Light rays

Electron beam

\section{Resolution}

\begin{tabular}{l|l} 
Below $0.25 \mu \mathrm{m}$ up to $0.30 \mu \mathrm{m}$ & Down to $0.4 \mathrm{~nm}$
\end{tabular}

\begin{tabular}{|l|l|}
\hline & \multicolumn{2}{|c|}{ Magnification/depth of field } \\
\hline 500x to 1500x & 2000000x \\
\hline Small & Large \\
\hline
\end{tabular}

Lenses material/vacuum

Optical glass/no need for vacuum Electromagnets/high vacuum is needed

\begin{tabular}{|l|l|l|l|}
\hline & \multicolumn{1}{|c|}{ Image dimension/colour } \\
\hline 2-D/3-D & 2-D/3-D \\
\hline RGB & Grey scale \\
\hline & Specimen preparation \\
\hline Simple & Difficult \\
\hline \multicolumn{2}{|c|}{ Applications } \\
\hline Live/dead specimens & Dead and dried specimens \\
\hline Cheap/negligible & Cost/maintenance \\
\hline \multicolumn{1}{|c|}{ Chemical composition: qualitative/quantitative analysis } \\
\hline Unavailable & $\begin{array}{l}\text { Available and accurate (the latter depends on } \\
\text { sample preparation and equipment operation) }\end{array}$ \\
\hline
\end{tabular}

reduction. On the other hand, lower voltage and high beam current necessary for EDS analysis and detection of certain elements are more problematic. ESEM is a conventional SEM that operates from lower pressures up to, at least, the pressure required to observe liquid distilled water. This microscope allows imaging of uncoated samples since accumulated electrical charges and even electrons from the electron beam are scattered by collision with the gas molecules inside the microscope chamber $[5,6]$.

p0055 Moreover, the combination of SEM imaging and elemental qualitative/ quantitative analysis given by EDS made it into one of the most powerful tools in scientific research. 


\section{s0020 1.2 Energy Dispersive X-Ray Spectroscopy}

p0060 X-rays are highly energetic photons resulting from electronic transitions in the atoms of a targeted solid by an incident accelerated electron beam. Typically, the incident electron hits an atom of the specimen and knocks out an electron from the K-shell ( $\mathrm{n}=1$ shell) of the metal, and a vacancy or hole is left in that shell. If an electron from another shell fills in that vacancy (electron transitions), then X-rays are emitted. Electronic transitions to the K-shell $(\mathrm{n}=1)$ are named $\mathrm{K}_{\mathrm{X} \text {-rays }}$, those to the L-shell $(\mathrm{n}=2)$ are $\mathrm{L}_{\mathrm{X} \text {-rays }}$ and to the M-shell $(n=3)$ are the $M_{X \text {-rays. These transitions are characteristic of each chemical }}$ element and this is the main reason that led to the development of EDS detection systems in electron microscopy and consequently widening its use in materials microstructural characterization. As mentioned earlier, the interaction volume and the volume from which the X-rays are generated are key factors for the quality of the final EDS spectrum. Moreover, the amount of generated signal strongly depends on the energy of the X-rays and the average atomic weight of the sample. For example, $\mathrm{X}$-rays such as carbon $\mathrm{K}_{\alpha}$ are easily absorbed by the solid sample and only a few are detected. In contrast, harder $\mathrm{X}$-rays such as iron $\mathrm{K}_{\alpha}$ are able to rupture the solid specimen and only a small amount is absorbed [5-7].

p0065 These characteristic X-rays are the analytical signals used in electron microscopy for chemical analysis. An X-ray spectrum emitted by the specimen provides both qualitative and quantitative information, allowing identification of which elements are present in the sample and the amount of each element. In addition to the emitted X-rays, a small amount of secondary X-rays can also be induced when the primary X-rays pass through the specimen and interact with the atoms of the sample [5-7].

p0070 The spatial resolution of X-ray microanalysis is the smallest distance between two volumes from which independent X-ray microanalyses are obtained. Since the interaction volume depends on the incident beam diameter as well as the spreading of the beam caused by elastic scattering of the beam within the sample, then microanalysis spatial resolution will strongly depend on the specimen. On the other hand, increasing spatial resolution compromises the limit of detection for a particular chemical element. The higher the spatial resolution, the smaller is the analysed volume and, consequently, the smaller is the signal intensity [5-7].

\section{S0025 2. SAMPLE PREPARATION}

p0075 Since SEM-EDS is sensitive enough for most purposes, sample derivatization is unnecessary, and the introduction of potential artefacts is avoided. Moreover, the minimum amount of sample required for observation offers the possibility to detect microplastics in environmental samples. For example, a single drop of a suspension placed onto the surface of a conductive plate/ support is more than sufficient for SEM observation. This enormous advantage 
enables characterization of several polymers with different size and morphology present in the same trial. Finally, some of the sample preparation techniques and methodologies used for observation of biological specimens under the electron beam are also quite useful for polymers, particularly for microplastics.

p0080 The preparation of specimens for examination in the SEM is the most important step for an effective imaging acquisition and accurate analytical analysis. The electron beam is highly energetic, and the yield of electrons hitting the specimen is higher than that leaving the specimen. Accordingly, there is an excess of electrons building up on the sample surface, and it is compulsory that the sample is electrically conductive. Moreover, the surplus of electrons needs to be grounded otherwise incoming primary electrons are repelled by the negatively charged surface and the image becomes distorted by the charging effects. In case of nonconductive samples such as ceramic materials, polymers or biological specimens, it is necessary to cover the surface of the sample, for example, with a very thin layer of carbon by high vacuum evaporation coating or gold or gold/palladium alloy or platinum thin film using a plasma sputter coater. Surface coating by vacuum is based on heating a conductive material to its vaporization temperature, followed by condensation of the evaporated atoms onto the surface of the sample, under high vacuum. In a typical plasma sputter coater, the metallic target is the cathode and the sample is placed on the anode electrode. The applied voltage between the electrodes produces plasma directed by a magnetron on the sample surface. In this way, the negatively charged conductive metal surface (placed on the cathode) is sputtered by the ionized gas and the sputtered metal coats the sample surface. In both methods the thickness of the conductive film is usually around $10 \mathrm{~nm}$, although variable, depending on the sample nature and its conductivity requirements. The film should not be too thick since it may mask the fine details of the surface or interfere with the other signals that may be detected such as X-ray emission. For example, if carbon is one of the elements of interest to be identified or eventually semiquantified, one should use a different conductive film to not interfere with EDS analysis. On the other hand, a too thin film may not be enough to prevent degradation by beam heating, radiation damage or specimen volatility. Since the EM is kept in high vacuum to avoid electron scattering and produce the electron beam as monochromatic as possible, the effect of specimen volatility is minimized to a certain point $[5,6,8]$.

p0085 Specimen preparation is an added critical factor in EDS because it will determine the depth of the $\mathrm{X}$-rays generation and range. For a correct quantitative X-ray analysis, the sample needs to be polished into a flat surface, minimizing height differences at interfaces and eliminating the geometric effects that arise from the specimen surface. For that, usually the sample is embedded in a resin block and polished with silicon carbide paper with different grids until the surface of the specimen is exposed. The latter is 
verified by OM. The polishing process requires careful inspection prior and after changing between very silicon carbide grids. If artefacts such as variable thickness or deceiving defects are introduced in the sample somewhere along the polishing progress, then distorted defect intensities and deformationinduced defects will be observed and those will be unreal features observed in the sample. Then, diamond cloths and paste are used for a finer and final polishing. Finally, conductive carbon or silver paint are used to attach the block onto a microscope specimen stub and, again, the surface becomes conductive by carbon or gold (or gold/palladium alloy) sputtering. As usual, a conducting path to earth needs to be provided after sample mounting, for example, using a conductive paint. There are many methods and several techniques that are applied in sample preparation for EM observation and analysis. Nonetheless, one should keep in mind that the best sample preparation method is most probably the less invasive one and the best sample is always the one that remains closest to its natural state [5-8].

p0090 A general sample preparation method for the specific case of microplastics analysis is now described. The support of the sample is very important since it should provide stability and security during handling and SEM-EDS observation. If the specimen is a dried soft or hard solid, it can be placed onto the surface of a conductive double-sided adhesive (aluminium or copper or silver or carbon, etc.) tape previously mounted onto a microscope specimen stub, or a conductive paste can also be used to fix the samples. Provided that the sample does not interact with its dispersion media, the same procedure may be applied if the microplastic is present in a suspension and a drop of the latter can be placed onto the surface of a conductive support and left to dry in air or under gentile vacuum. In the latter case, and unless a solvent free paste is used, the sample dispersion medium must not react with the conductive paste or tape. For example, if the dispersion medium of the sample is acetone then it will dissolve the glue of the tape or the dilute/unstiffen the conductive paste (water, alcohol or acetone based). Alternatively, a small piece of the microplastic can be embedded in a liquid resin which is later polymerized to a solid and sectioned or polished (silicon carbide paper followed by diamond suspensions) to reveal the embedded microplastic. Then, the sample needs to be stabilized into minimize radiation effects, improve electric conductivity and signal-to-noise ratio. Similarly, for biological specimens, microplastics are composed of low atomic number elements exhibiting a very low signal-tonoise ratio. The contrast of the sample may be enhanced by staining it (chemically or physically incorporated into the microplastic matrix) with high atomic number compounds such as chlorosulphonic or phosphotungstic acid, ebonite, osmium or ruthenium tetroxide and silver salts (sulphide and nitrate) [8]. After sample immobilization, the essential electrical and thermal conductivity of the sample are attained by coating its surface with a very thin layer of a conductive metal such as carbon thin film using a high vacuum evaporator coater. To provide the most stability to the sample, bearing in mind 
that surface masking or artefacts must be avoided, a slightly thicker than $10 \mathrm{~nm}$ thin film is deposited onto the surface of the sample.

p0095 Fig. 2 illustrates the surface morphology observed using the SEM, at $5 \mathrm{kV}$, of common thermoplastics such as polyethylene (PE), polypropylene (PP), polystyrene (PS) and polyvinyl chloride (PVC).

\section{S0030 3. EQUIPMENT OPERATION}

p0100 A sample unclean and unstable under the electron beam can induce contamination, beam damage or specimen drift, which are factors that imperatively should be minimized into attain imaging and microanalysis with the best quality. In the previous section, it has been mentioned that proper sample preparation is the first step to practise correct electron microscopy and consequent accurate analysis. Likewise, equipment settings and operation also need to be addressed since the operator adopted procedure critically influences the final resulting images and analytical analysis. Polymers are damaged by ionization radiation exposure and thermal effects. The effects cause chemical bonds breaking, mass loss, reduction of crystallinity and creation of volatile material in the SEM high vacuum. The latter can be reduced if one uses the minimum beam energy needed to provide the maximum information. Fig. 3
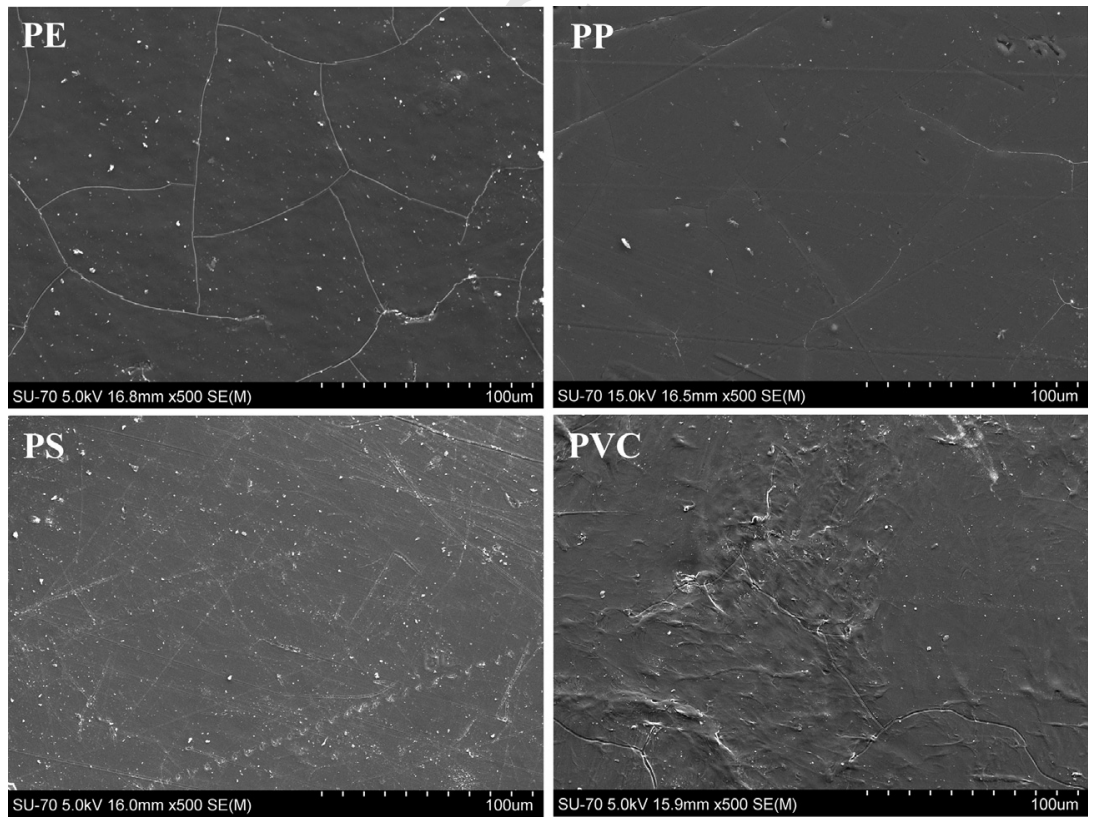

f0015 FIGURE 2 Scanning electron microscopy images showing the surface of several thermoplastics: polyethylene, polypropylene, polystyrene and polyvinyl chloride. 

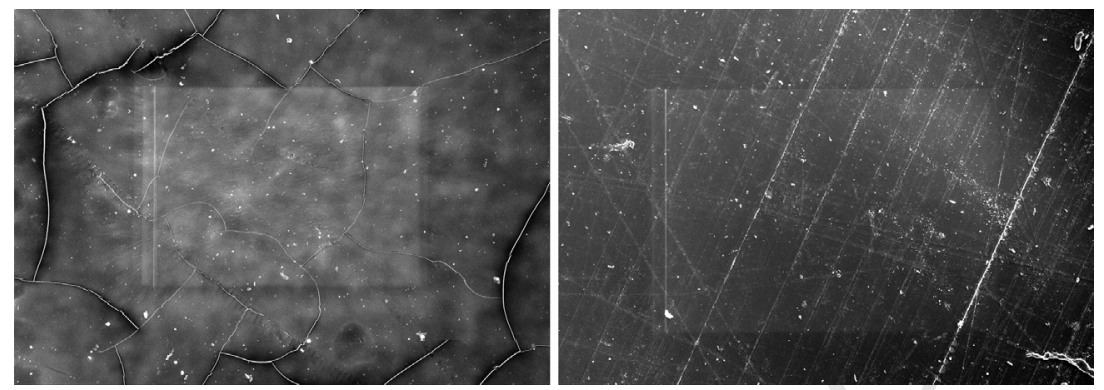

f0020 FIGURE 3 Scanning electron microscopy images showing examples of volatile material deposition onto the surface of polyethylene and polypropylene caused by electron beam damage.

shows an example of volatile material deposition onto the surface of polyethylene and polypropylene caused by electron beam damage.

p0105 Ideally, the lowest voltage and beam current should be the working conditions, but imaging and elemental analysis may be compromised. Therefore, it will always depend on the objectives of the SEM usage on a certain specimen. In the case of a microplastic analysis, if the sample is properly prepared, a relatively common acceleration voltage like $15 \mathrm{kV}$ may be applied. Nevertheless, the microscope operator will easily see that there are limitations in imaging at such operation conditions. For example, there will be a limit in the magnification used for imaging purposes due to damage induced by the electron beam, as Fig. 4 demonstrates.

p0110 Moreover, careful inspection of the microplastic must be carried out since some details on the surface of the microplastic may have been caused by exposure to the electron beam and can easily be mistaken for fine features of

[Q4] the microstructure, as shown in the next figure (Fig. 5).

p0115 Considering EDS analysis, some attention should be given to the time allowed for the pulse processor to evaluate the pulse magnitude (time constant) as well as the dead time. The latter is the time it takes from the arrival of a
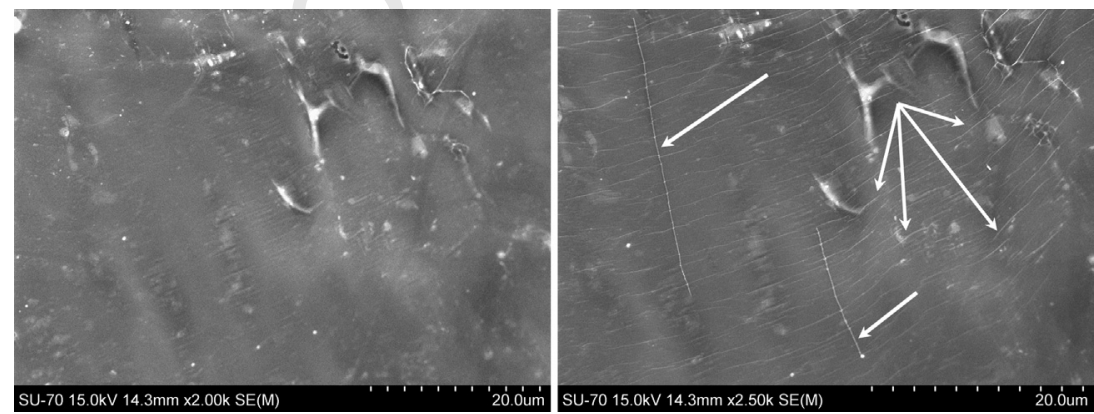

f0025 FIGURE 4 Scanning electron microscopy images showing the surface of a microplastic before and after increasing magnification causing electron beam damage. 

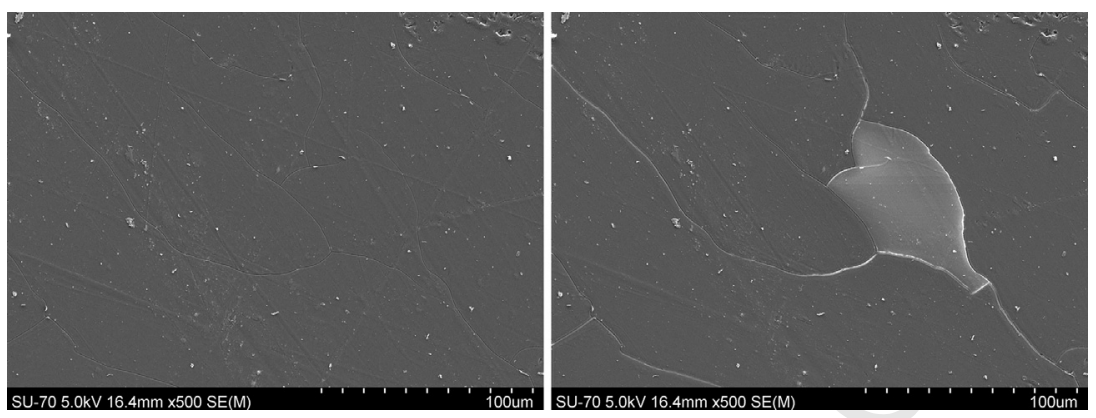

f0030 FIGURE 5 Scanning electron microscopy (SEM) images showing the surface of a microplastic before and after being exposed for $5 \mathrm{~min}$ to the SEM electron beam operated at $5 \mathrm{kV}$.

pulse that automatically switches off the detector to the end of analysis by the pulse processor. This is highly relevant since there has to be an equilibrium between the best possible energy resolution and the count rate (number of counts per second, cps). Depending on the applied accelerating voltage and if using a $\mathrm{Si}(\mathrm{Li})$ detector, a typical selectable energy range is $20 \mathrm{keV}$ with a resolution around $10 \mathrm{eV}$ per channel. The detector collection angle is the solid angle subtended at the analysis point on the specimen by the active area of the front face of the detector. Usually, the detector is fixed in the microscope, already tilted towards the specimen and placed as close as possible to it. Nevertheless, attention needs to be given when the specimen may need additional rotation of the specimen to face the detector. Further tilting of the sample may be necessary or avoided since it will critically influence the final analytical analysis, particularly the quantitative one [7].

p0120 EDS spectra acquisition and data treatment aiming qualitative and quantitative analysis require particular consideration. Firstly, the operating conditions should be adjusted to maximize the X-rays count rate at the possible highest accelerating voltage to avoid damage of beam-sensitive specimens like microplastics. The spectrum should be collected from large areas, using large probe (combination of probe size and final aperture) and for an optimized period of time (change in chemical composition or contamination may take place for long spectrum acquisition times). If needed, further analysis of smaller areas can be carried out under conditions in which the spatial resolution is improved. For representative purposes and statistical confidence, as well as to secure that a light chemical element present in a few weight percent is also detected, several spectra in random areas of the sample should be collected. After spectra acquisition, the peaks need to be assigned and that is, easily achieved if the most intense peak and working down through

[Q5] its family is identified. Then, the next most intense peak (not included in the previous family) is also assigned, and pathological overlaps may occur and should be deconvoluted. The process is repeated until all the observed peaks are identified. At the present time, most EDS software systems are user 
friendly and make things easier for peak assignment. As previously pointed out, quantitative SEM-EDS should only be carried out in flat polished samples or very thin films with irrelevant topography. Prior to spectra acquisition for quantification purpose, it is imperative that several well-assigned spectra with sufficient X-ray counts have been previously collected. Then, the spectra need to be processed with matrix corrections with oxygen calculated by stoichiometry and the appropriate ZAF (the atomic number-Z, absorption-A and fluorescence-F correction) procedure. Again, EDS software applies the ZAF correction and carries out subsequent integration of the peaks to give the final semiquantification of the elements present in the specimen. Nevertheless, the user should be as critical as possible when accepting each step of the quantification process provided by the software [7].

\section{S0035 4. MICROPLASTICS CHARACTERIZATION}

p0125 Most of the few published works involving SEM-EDS and microplastics were carried out to identify and determine the morphology of typical microplastic fragments, especially when present in marine environments. To the best of our knowledge, SEM-EDS has only started to be applied in microplastic research during this decade. For example, Hidalgo-Ruz et al. [9] published in 2012 a review paper regarding methods used for identification and quantification of microplastics in the marine environment, and SEM-EDS was not even contemplated. Von der Kammer et al. [10] published another critical review about the identification and analysis of micro/nanomaterials, including polymer-based materials present in complex matrices, in which the potentiality of EM is finally acknowledged.

p0130 SEM application has been a tool especially used on the study of microplastics present in water media. Van Cauwenberghe et al. [11] showed, for the first time, that microplastics are present in the top sediment layer of the deepsea floor dreading that microplastics most probably have spread throughout all the seas and oceans, including the remote and largely unknown deep sea. Moreover, Erikssen et al. [12] found the presence of microplastics in surface samples collected from the Laurentian Great Lakes. Weinstein et al. [13] demonstrated that UV radiation may also play a role in the degradation of plastic debris into microplastics. SEM-EDS was a practical technique in the identification of inorganic plastic additives contained in microplastic particles and in the characterization of microplastics and toxic chemicals extracted from the microparticles in samples from the North Pacific Gyre [14,15].

p0135 The interaction between biota and the microplastic particles present in water media has also become a research target. Carson et al. [16] investigated the plastic-associated microorganisms of the North Pacific Gyre and used SEM imaging to finding occasional bacterial presence which increased on items with rough surfaces, at sites with high plastic concentrations, including foamed polystyrene. Imhof et al. [17] identified degraded microplastics in 
beach sediments of a subalpine lake using SEM imaging. Many of the polypropylene microplastics showed cracks, pits, grooves, disintegration, and adherent and attached diatoms. Equally, Zettler et al. [18] also investigated microbial communities on plastic marine debris finding that the latter are mainly a novel ecological habitat to a diverse microbial community of heterotrophs, autotrophs, predators and symbionts, which the authors refer to as Plastisphere. Further studies have been carried out considering the interaction between these emergent pollutants and biota by SEM imaging [19-21]. All the authors concluded that microplastics have been used as habitats for rapid bacteria colonization. Consequently, it is imperative that this occurrence and consequent ecological implications are determined as well as minimized for marine organism distribution, ocean yield and biotransfer of plasticassociated pollutants.

p0140 ESEM has also been used in the identification of microplastic particles in sediments of Lagoon of Venice (Italy). Vianello et al. [22] analysed sediments using several techniques and an ESEM $(25 \mathrm{kV})$ to characterize the surface morphology of the microplastic particles. ESEM showed irregular shapes and similar surface textures for several microplastics in contrast with comparable amorphous and regular surfaces of other plastic microfibers; it also showed weakened surfaces caused by adhering particles probably indicating the occurrence of degradation processes.

p0145 Regarding transmission electron microscopy (TEM), and since the electron beam is usually operated at an acceleration voltage of 80,200 or $300 \mathrm{kV}$, it makes it nearly impossible to observe nanosized plastics without destroying them immediately under the highly energetic electron beam or the prior need for more sophisticated techniques to prepare the sample and, most probably, changing it or introducing several artefacts. All the same, a recent study using a low-voltage TEM $(5 \mathrm{kV})$ has been performed on the analysis of nanosized plastics: Gigault et al. [23] reported on the resulting nanoplastics, under $100 \mathrm{~nm}$, in size due to the UV degradation of microplastic fragments present in samples collected at the North Atlantic Ocean. The results demonstrated the potential hazards of nanoplastic wastes have not yet been taken properly into account. Booth et al. [24,25] studied both polymethylmethacrylate-based (PMMA) nanoplastics synthesis and their uptake and toxicity on different aquatic organisms. Nanoparticle size was assessed by conventional TEM inspection revealing spherical particles with a broad dimension between 86 and $125 \mathrm{~nm}$. The data indicated that the ecotoxicity of PMMA nanoparticles could not be reliably estimated using a single nanoplastic type. Besseling et al. [26] tested polystyrene nanoparticles $(\sim 70 \mathrm{~nm})$ on growth and reproduction of different organisms. The study pointed out a possible synergistic effect of the nanoplastics together with the traditional factors of stress related to aquatic organisms. Recent and significant reviews have been published considering the presence of nanoplastics in the environment as well as their sources, fates and effects [27,28]. 
p0150 As a final note, atomic force microscopy (AFM) has not yet been explored in topography determination of microplastics. Combining the advantages of high resolution imaging with the ability of being operated in liquid environment and not requiring a conductive sample, AFM is most probably a quite valuable tool for imaging microplastics in liquid matrices. As with any other characterization technique, the main drawbacks are related to a less feasible change of magnification and still there is no implemented system of elemental analysis.

p0155 In conclusion, SEM-EDS has become an indispensable tool for the identification and characterization of microplastic particles. This powerful technique enabled researchers to conclude that early stages of plastic debris degradation proceed fairly rapidly to the production of microplastic particles, emergent pollutants expressively threatening biota life.

\section{S0040 5. FINAL CONSIDERATIONS}

p0160 SEM-EDS is a highly specialized technique largely used in the characterization of micro- and nanomaterials. Its application has generally been extended to other science areas such as biology. In the last few years, it has also been used in the assessment of the morphology and chemical nature of polymerbased materials such as microplastics. This is a direct result arising from the need of further and better understanding of microplastics as an environmental pollutant. Moreover, the fact that human activities continuously introduce micro-/nanosized substances into the environment stressed the demand of dedicated techniques.

p0165 This chapter highlighted the role of electron microscopy coupled with analytical analysis, particularly SEM-EDS, in the characterization of microplastics, with particular emphasis on their identification and morphology determination. A general appreciation concerning sample preparation and equipment operation is also given. The rising relevance of such advanced technique has been clearly confirmed and its increasing use in future studies regarding characterization of microplastics and their influence as emergent pollutants is more than expected, as well as indispensable.

\section{REFERENCES}

[1] W.J. Croft, Under the Microscope: A Brief History of Microscopy, World Scientific Publishing Co. Pte. Ltd., Singapore, 2006.

[2] R. Haynes, Optical Microscopy of Materials, Springer Science + Business LLC, Media New York, New York, 1984.

[3] P. Török, F. Kao, Optical Imaging and Microscopy - Techniques and Advanced Systems, second ed., Springer-Verlag Berlin Heidelberg, New York, 2007.

[4] R.F. Egerton, Physical Principles of Electron Microscopy - An Introduction to TEM, SEM, and AEM, Springer Science + Business Media, Inc., New York, 2005. 
[5] P.J. Goodhew, J. Humphreys, R. Beanland, Electron Microscopy and Analysis, third ed., Taylor \& Francis, New York, 2001.

[6] V. Kazmiruk, Scanning Electron Microscopy, InTech, Rijeka, 2012.

[7] A.J. Garratt-Reed, D.C. Bell, Energy Dispersive X-Ray Analysis in the Electron Microscope, BIOS Scientific Publishers Limited, Oxford, 2003.

[8] P. Echlin, Handbook of Sample Preparation for Scanning Electron Microscopy and X-Ray Microanalysis, Springer Science + Business LLC, Media New York, New York, 2009.

[9] V. Hidalgo-Ruz, L. Gutow, R.C. Thompson, M. Thiel, Microplastics in the marine environment: a review of the methods used for identification and quantification, Environ. Sci. Technol. 46 (2012) 3060-3075.

[10] F. von der Kammer, P.L. Ferguson, P.A. Holden, A. Masion, K.R. Rogers, S.J. Klaine, A.A. Koelmans, N. Horne, J.M. Unrine, Analysis of engineered nanomaterials in complex matrices (environment and biota): general considerations and conceptual case studies, Environ. Toxicol. Chem. 31 (2012) 32-49.

[11] L. van Cauwenbergue, A. Vanreusel, J. Mees, C.R. Janssen, Microplastic pollution in deepsea sediments, Environ. Pollut. 182 (2013) 495-499.

[12] M. Eriksen, S. Mason, S. Wilson, C. Box, A. Zellers, W. Edwards, H. Farley, S. Amato, Microplastic pollution in the surface waters of the Laurentian great lakes, Mar. Pollut. Bull. 77 (2013) 177-182.

[13] J.E. Weinstein, B.K. Crocker, A.D. Gray, From macroplastic to microplastic: degradation of high-density polyethylene, polypropylene, and polystyrene in a salt marsh habitat, Environ. Toxicol. Chem. 35 (2016) 1632-1640.

[14] E. Fries, J.H. Dekiff, J. Willmeyer, M. Nuelle, M. Ebert, D. Remy, Identification of polymer types and additives in marine microplastic particles using pyrolysis-GC/MS and scanning electron microscopy, Environ. Sci. Process. Impacts 15 (2013) 1949-1956.

[15] L.M.R. Mendoza, P.R. Jones, Characterisation of microplastics and toxic chemicals extracted from microplastic samples from the North Pacific Gyre, Environ. Chem. 12 (2015) 611-617.

[16] H.S. Carson, M.S. Nerheim, K.A. Carroll, M. Eriksen, The plastic-associated microorganisms of the North Pacific Gyre, Mar. Pollut. Bull. 75 (2013) 126-132.

[17] H.K. Imhof, N.P. Ivleva, J. Schmid, R. Niessner, C. Laforsch, Contamination of beach sediments of a subalpine lake with microplastic particles, Curr. Biol. 23 (2013) R867-R868.

[18] E.R. Zettler, T.J. Mincer, L.A. Amaral-Zettler, Life in the "plastisphere": microbial communities on plastic marine debris, Environ. Sci. Technol. 47 (2013) 7137-7146.

[19] J.P. Harrison, M. Schratzberger, M. Sapp, A.M. Osborn, Rapid bacterial colonization of lowdensity polyethylene microplastics in coastal sediment microcosms, BMC Microbiol. 14 (2014) 232.

[20] S. Oberbeckmann, M.G.J. Loeder, G. Gerdts, A.M. Osborn, Spatial and seasonal variation in diversity and structure of microbial biofilms on marine plastics in Northern European waters, FEMS Microbiol. Ecol. 90 (2014) 478-492.

[21] J. Reisser, J. Shaw, G. Hallegraeff, M. Proietti, D.K.A. Barnes, M. Thums, C. Wilcox, B.D. Hardesty, C. Pattiaratchi, Millimeter-sized marine plastics: a new pelagic habitat for microorganisms and invertebrates, PLoS One 9 (2014) e100289.

[22] A. Vianello, A. Boldrin, P. Guerriero, V. Moschino, R. Rella, A. Sturaro, L. da Ros, Microplastic particles in sediments of Lagoon of Venice, Italy: first observations on occurrence, spatial patterns and identification, Estuar. Coast Mar. Sci. 130 (2013) 54-61. 
16 Comprehensive Analytical Chemistry

[23] J. Gigault, B. Pedrono, B. Maxit, A. Ter Halle, Marine plastic litters: the unanalyzed nanofraction, Environ. Sci. Nano 3 (2016) 346-350.

[24] A.M. Booth, J. Justynska, S. Kubowicz, H. Johnsen, M. Frenzel, Influence of salinity, dissolved organic carbon and particle chemistry on the aggregation behaviour of methacrylate-based polymeric nanoparticles in aqueous environments, Int. J. Environ. Pollut. 52 (2013) 15-30.

[25] A.M. Booth, B.H. Hansen, M. Frenzel, H. Johnsen, D. Altin, Uptake and toxicity of methylmethacrylate-based nanoplastic particles in aquatic organisms, Environ. Toxicol. Chem. 35 (2016) 1641-1649.

[26] E. Besseling, A. Wegner, E.M. Foekema, M.J. van den Heuvel-Greve, A.A. Koelmans, Environ. Sci. Technol. 47 (2013) 593-600.

[27] A.A. Andrady, Microplastics in the marine environment, Mar. Pollut. Bull. 62 (2011) $1596-1605$.

[28] J.P. da Costa, P.S.M. Santos, A.C. Duarte, T. Rocha-Santos, (Nano)plastics in the environment - sources, fates and effects, Sci. Total Environ. 566-567 (2016) 15-26. 